\title{
Inhaled Magnesium in Acute Bronchiolitis: Another One Bites the Dust?
}

\author{
Joseph L. Mathew ${ }^{1}$
}

Received: 6 September 2021 / Accepted: 10 September 2021 / Published online: 17 September 2021

(c) Dr. K C Chaudhuri Foundation 2021

Bronchiolitis is one of the most vexing clinical challenges faced by pediatricians worldwide. On the one hand, it is the most common acute respiratory illness affecting young infants, the majority of whom recover uneventfully with supportive care alone. On the other hand, it can sometimes result in severe illness with life-threatening complications, wherein nothing seems to work. The authoritative guidelines published by the National Institute of Health and Clinical Excellence (NICE), UK (that were updated as recently as August 2021), emphatically recommend against using various commonly tried therapies including nebulized epinephrine, hypertonic saline, salbutamol, ipratropium, corticosteroids (inhaled or systemic), leukotriene receptor antagonists, antibiotics, and combinations of some of these [1]. Despite immense research, the wise adage enunciated almost $60 \mathrm{y}$ back, that "oxygen therapy is vitally important in bronchiolitis and there is little convincing evidence that any other therapy is consistently or even occasionally useful" [2] accurately sums up the therapeutic options available even today. The helplessness and hopelessness faced by clinicians and researchers led some investigators to experiment with magnesium sulphate in bronchiolitis, on the grounds that it works in bronchial asthma.

This issue of the Journal reports a randomized controlled trial (RCT) comparing inhaled magnesium sulphate versus standard treatment alone, in bronchiolitis [3]. The trial included 60 infants 1-24 mo old, hospitalized with a clinical diagnosis of moderate-to-severe bronchiolitis. All received hydration and humidified oxygen (although the details were not described). In addition, they received either iso-osmolar $3.2 \%$ magnesium sulphate ( 6 doses at 4 hourly intervals), or nothing. Multiple meticulously recorded outcomes failed to show any benefit of magnesium in terms of, bronchiolitis

Joseph L. Mathew

dr.joseph.1.mathew@gmail.com

1 Department of Pediatrics, Advanced Pediatrics Centre, PGIMER, Chandigarh 160012, India severity score at various time-points within the first $24 \mathrm{~h}$, duration of hospitalization, duration of oxygen therapy, escalation of therapy (respiratory support, intensive care), and postdischarge need for medical care (out-patient, in-patient, or ICU) within $14 \mathrm{~d}$. There were no adverse events observed, although it is unclear what was specifically looked for (other than 'acute cardiorespiratory instability').

The trial [3] was appropriately registered [4], with minor (beneficial) deviations from the stated plan (i.e., reporting of three unplanned outcomes viz. duration of hospitalization, need for noninvasive ventilation, and duration of oxygen therapy). Suitable methods were used to generate the randomization sequence and achieve concealment of allocation. However, there was no blinding. There was no apparent bias related to selective or incomplete data reporting. Clinically relevant, patient-centric outcomes were assessed. Overall, the trial had low to moderate risk of bias.

The investigators [3] did not use saline as a placebo in the comparison arm. In a sense, this is fortuitous because recently, there has been debate whether saline itself has clinical effects in bronchiolitis [5]. Therefore, had the investigators reported comparable effects using saline as a placebo, the results would have been harder to interpret. Blinding of the outcome assessor should have been seriously considered given that many of the outcomes measured are prone to assessor bias. The four-criteria bronchiolitis severity score includes respiratory rate, wheezing, chest retraction, and general condition, the last of which is susceptible to variable interpretations. Of course, it is an entirely different matter that none of the clinical scores are themselves very robust [6]. As in routine practice in most Indian institutions, the investigators [3] did not undertake virology studies to look for RSV.

There is very scanty evidence on inhaled magnesium in bronchiolitis. A Turkish three-arm trial in 57 infants compared nebulized magnesium, combination of salbutamol plus magnesium, and saline alone [7]. Although there were no statistically or clinically significant differences in length of 
hospitalization, the clinical severity score appeared to be better in those who received the combination of salbutamol and magnesium. However, the trial had high risk of bias.

Another trial in Iran among 125 infants compared inhaled magnesium plus epinephrine (in normal saline) vs. epinephrine alone [8]. There was no benefit of the combination in terms of length of hospitalization, duration of oxygen therapy, and escalation to respiratory support. However, there was statistically significant benefit in clinical severity score on the second and third days, although the clinical significance is questionable, especially considering the absence of any difference within the first $24 \mathrm{~h}$. Overall, this trial had low risk of bias.

An unpublished Indian trial comparing inhaled magnesium vs. hypertonic saline showed comparable results. Although this trial is not comparable to the others $[3,7,8]$ because magnesium was not compared to placebo/no treatment, it is the largest of these trials (220 infants), and it provides valuable information on baseline characteristics and outcomes in the infants.

Despite the paucity of data, inhaled magnesium has already featured in two systematic reviews. The first of these published in 2017 [9] suggested that magnesium may be helpful, but the review is outdated. More recently, a Cochrane review published in December 2020 [10], identified the trials cited above [7, 8], and concluded that there is insufficient evidence on efficacy and/or safety of inhaled magnesium in bronchiolitis, recommending (as usual) welldesigned clinical trials. In that context, the latest trial [3] is a valuable addition to literature. However, the diversity of the trials $[3,7,8]$ in terms of participants and interventions makes it inappropriate to update the meta-analysis, despite the fact that the trials used similar outcome measures.

In summary, at present it appears that inhaled magnesium offers little hope in the dismal therapeutic armamentarium to treat acute bronchiolitis. Paraphrasing the lyrics of the iconic song by Queen: "Another one (intervention in bronchiolitis) bites the dust."

\section{Declarations}

Conflict of Interest None.

\section{References}

1. Bronchiolitis in children: diagnosis and management. In: NICE guideline [NG9]. 2015. Available at: https://www.nice.org.uk/ guidance/ng9/chapter/1-Recommendations\#management-ofbronchiolitis. Accessed on 5 Sep 2021.

2. Reynolds EO, Cook CD. The treatment of Bronchiolitis. J Pediatr. 1963;63:1205-7.

3. Debbarma R, Khera D, Singh S, Toteja N, Choudhary B, Singh K. Nebulized magnesium sulphate in bronchiolitis: a randomized controlled trial. Indian J Pediatr. 2021:1-6. https://doi.org/10. 1007/s12098-021-03695-8.

4. Khera D. Role of Magnesium Sulfate in children having Bronchiolitis. Available at: http://ctri.nic.in/Clinicaltrials/advsearch.php. Accessed on 5 Sep 2021.

5. Mathew JL. Does normal saline have clinical effects in infants with bronchiolitis?: Evidence-based Medicine Viewpoint. Indian Pediatr. 2020;57:254-7.

6. Bush A, Nagakumar P. Acute bronchiolitis: still no new treatments to offer. Indian J Pediatr. 2015;82:777-8.

7. Kose M, Ozturk MA, Poyrazoğlu H, et al. The efficacy of nebulized salbutamol, magnesium sulfate, and salbutamol/magnesium sulfate combination in moderate bronchiolitis. Eur J Pediatr. 2014;173:1157-60.

8. Modaresi MR, Faghihinia J, Kelishadi R, et al. Nebulized magnesium sulfate in acute bronchiolitis: A randomized controlled trial. Indian J Pediatr. 2015;82:794-8.

9. Kua KP, Lee SW. Complementary and alternative medicine for the treatment of bronchiolitis in infants: a systematic review. PLoS One. 2017;12:e0172289.

10. Chandelia S, Kumar D, Chadha N, Jaiswal N. Magnesium sulphate for treating acute bronchiolitis in children up to two years of age. Cochrane Database Syst Rev. 2020;12:CD012965.

Publisher's Note Springer Nature remains neutral with regard to jurisdictional claims in published maps and institutional affiliations. 